\title{
SUGGESTION OF A NEW CRITERION OF DYNAMIC FRACTURE INITIATION
}

Ya-PuZhao

Lab for Non-Linear Mechanics of Continuous Media, Institute for Mechanics Chinese Academy of Sciences, Beijing 100080, People's Republic of China Tel: (010) 2561834

Dynamic fracture is a time-dependent phenomenon, it is generally agreed that brittle dynamic fracture requires the dynamic stress intensity factor to exceed the dynamic fracture toughness for a certain minimum time [1-3]. The experimental and theoretical study of the dynamic initiation of 4340 VAR steel by plate impact tests with the same duration of $1 \mu s$ showed that the dynamic initiation process is dependent on the meso characteristic dimension of the material, namely, the interparticle spacing [4]. A sharp spike with a duration of less than 80 nanoseconds of the normal velocity at the rear surface of the specimen was observed by Prakash, Freund and Clifton [4] and this phenomenon was related to the onset of the crack growth. They modeled the crack initiation as the sudden formation of a very small hole at the crack tip, the radius of the hole, $\epsilon$, agrees reasonably well with the interparticle spacing. This admits the possibility of dynamic crack-tip stress fields with crack-tip singularities stronger $\left(\sim r^{3 n}\right)$ than the inverse square root singular fields of fracture mechanics.

Tuler and Butcher [5] suggested a famous criterion for dynamic fracture

$$
\int\left(\sigma-\sigma_{0}\right)^{\lambda} d t=K
$$

where $\sigma_{0}$ might be assigned the value of the dynamic yield point or the static true stress for fracture. $\lambda$ and $K$ are constants. Obviously, $\left(\sigma-\sigma_{0}\right)$ is the overstress. In accordance with the 'least action criterion' proposed by Steverding and Lehnigk $[6,7]$ for dynamic fracture and for convenience, here $\lambda=2$ is chosen.

By dimensional analysis, a new dynamic fracture criterion which is dimensionally equivalent to (1) is suggested as the following

$$
\int_{t_{0}}^{t_{1}} \frac{\left(K-K_{I d}\right)^{2}}{\epsilon} d t=C
$$

where $\dot{K}_{\mathrm{ld}}\left(K_{\mathrm{p}} T\right)$ is the dynamic fracture toughness which is a function with respect to both loading rate $\dot{K}_{\mathrm{I}}$ and temperature $T$, and $\left(K-K_{\mathrm{Id}}\right)$ is termed as the overstress 
intensity factor. Similar to [4], $\epsilon$ is a meso characteristic dimension which can be taken as the interparticle spacing. $C$ is a material constant, and $t_{0}$ is the time that $K=K_{\mathrm{Ld}}$.

For other values of $\lambda$, the corresponding criterion is expressed by

$$
\int_{t_{0}}^{t_{1}} \frac{\left(K-K_{l d}\right)^{\lambda}}{\epsilon^{\lambda / 2}} d t=C
$$

Acknowledgement: The support from the National Natural Science Foundation of China is acknowledged.

[1] J.F. Kalthoff and D.A. Shockey, Journal of Applied Physics 48 (1977) 986-993.

[2] D.A. Shockey, J.F. Kalthoff and D.C. Erlich, International Journal of Fracture 22 (1983) 217-229.

[3] J.F. Kalthoff, D.A. Shockey and H. Homma, Proceeding of 3 rd Conference on the Mechanical Property under High Rates of Strain, Oxford (1984) 205-211.

[4] V. Prakash, L.B. Freund and R.J. Clifton, Journal of Applied Mechanics, Transactions of ASME 59 (1992) 356-365.

[5] F.R. Tuler and B.M. Butcher, International Journal of Fracture Mechanics 4 (1968) 431-437.

[6] B. Steverding and S.H. Lehnigh, Journal of Applied Physics 41 (1970) 2096-2099.

[7] Ibid, 42 (1971) 3231-3238.

5 May 1995 